\title{
Pituitary stalk thickening: the role of an innovative MRI imaging analysis which may assist in determining clinical management
}

\author{
Emilia Sbardella1,3, Robin N Joseph2, Bahram Jafar-Mohammadi', \\ Andrea M Isidori ${ }^{3}$, Simon Cudlip ${ }^{4}$ and Ashley B Grossman ${ }^{1}$ \\ 'Department of Endocrinology, Oxford Centre for Diabetes, Endocrinology and Metabolism, Churchill \\ Hospital, University of Oxford, Oxford, UK, '2Department of Neuroradiology, John Radcliffe Hospital, \\ University of Oxford, Oxford, UK, ${ }^{3}$ Department of Experimental Medicine, Sapienza University of Rome, \\ Rome, Italy, and ${ }^{4}$ Department of Neurological Surgery, John Radcliffe Hospital, University of Oxford, \\ Oxford, UK
}

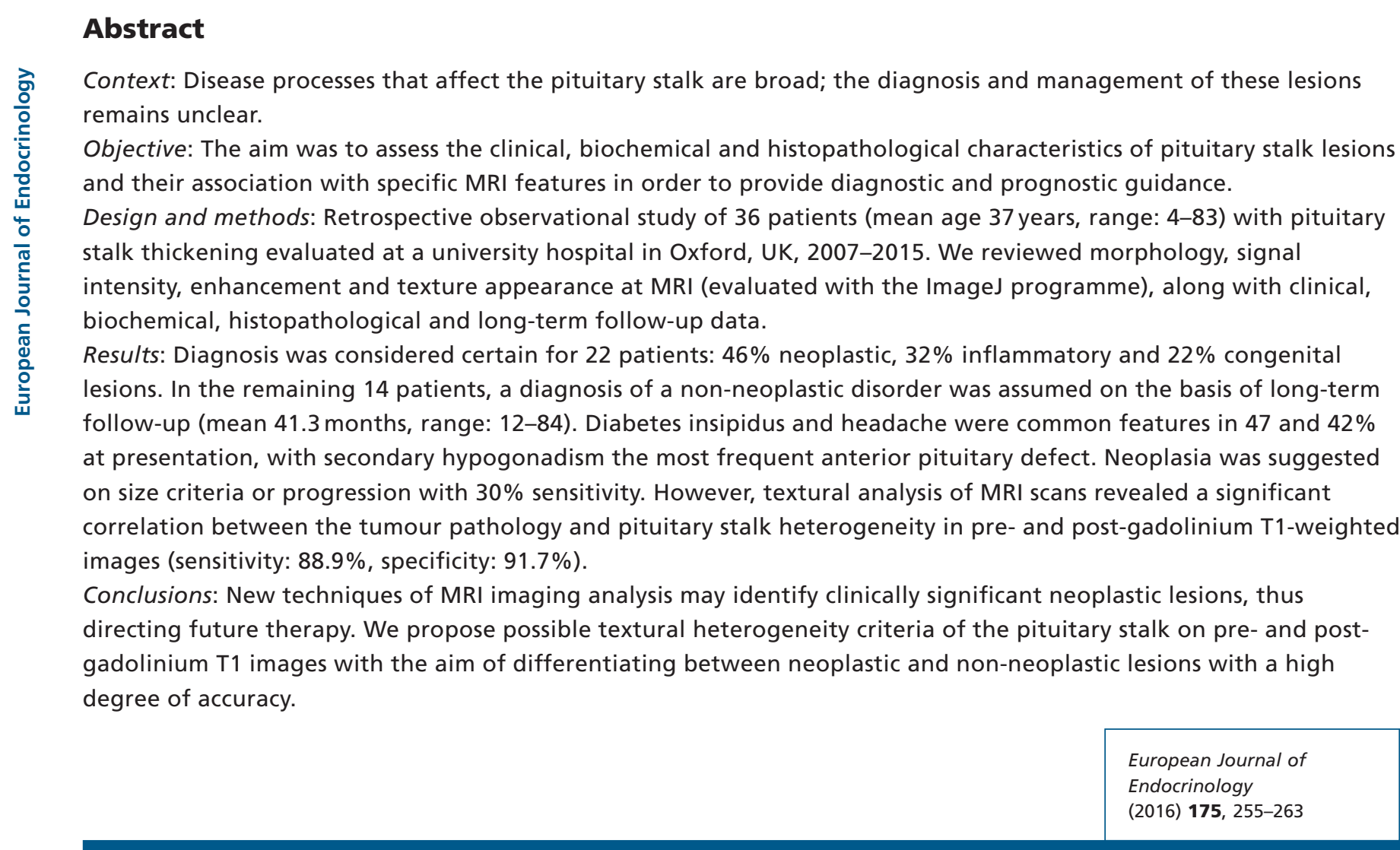

\section{Introduction}

The pituitary stalk connects the median eminence of the hypothalamus to the pituitary gland. Disease processes primarily involving the pituitary stalk present a demanding challenge for the clinician, given the relatively few specific signs of the disease and the problems in obtaining a

www.eje-online.org DOI: 10.1530/EJE-16-0455 (c) 2016 European Society of Endocrinology Printed in Great Britain tissue sample. The management of disorders associated specifically with abnormalities of the pituitary stalk is highly problematic, and it remains unclear as to how and when surgical intervention is required, given that surgery can be associated with significant morbidity. Magnetic 
resonance imaging (MRI) has proved to be an essential tool in the evaluation of intracranial pathology and of the majority of lesions affecting the hypothalamo-pituitary region $(1,2,3,4,5,6)$, but its role in determining when surgery is appropriate in this situation is controversial $(7,8)$.

The upper limit of normal of the width of the pituitary stalk is $3.25 \mathrm{~mm}$ near the median eminence and $1.9 \mathrm{~mm}$ at its insertion into the pituitary gland (9). Pituitary stalk lesions are often discovered on MRI either incidentally or during evaluation for symptoms related to various degrees of hypothalamic and/or pituitary dysfunction, especially in patients with isolated diabetes insipidus (DI). The differential diagnosis of masses affecting the pituitary stalk is wide, and can be grouped into three categories: congenital, inflammatory/infectious and neoplastic $(8,10,11,12)$. However, to obtain a definitive histological diagnosis would require biopsy of the stalk, which is a difficult procedure associated with considerable morbidity (13). Guidelines regarding the clinical management of such lesions are sparse, other than a recent review from the Mayo Clinic (6), and there are few centres with the large patient numbers and expertise to collate sufficient data on their management.

We have, therefore, conducted a retrospective analysis of patients with pituitary stalk thickening evaluated at a large referral centre over the last decade. In particular, we have tested whether novel analysis of MRI imaging can offer a diagnostic advantage and guide management of this rare condition.

\section{Patients and methods}

We identified patients with pituitary stalk thickening by an electronic and manual search of the weekly pituitary surgical multidisciplinary group records, the neurosurgical operation lists of the neurosurgeon (SC) performing this procedure and our pituitary patient database; the text words 'pituitary stalk' and 'infundibulum' were used in a computerised search.

Inclusion criteria were age between 4 and 80 years, and a cranial/pituitary MRI performed between January 2007 and December 2015. Exclusion criteria were patients with inadequate imaging or a normal pituitary stalk at image revision, and patients with pituitary or brain radiation/ surgery before the diagnostic MRI.

\section{Endocrine assessment}

The following data were collected from medical records: anthropometric parameters, signs and symptoms of DI or isolated/multiple anterior pituitary hormone deficiencies. Baseline endocrine tests including serum GH, IGF1, LH, FSH, testosterone or estradiol, prolactin, thyroid function, 09:00 h cortisol, plasma ACTH and dynamic function tests where available. Serum/CSF tumour and inflammatory markers such as hCG, $\alpha \mathrm{FP}$ and ACE were recorded when performed at any point either at presentation or during the course of the disease process.

\section{MRI evaluation}

All retrieved MRI scans were fully re-analysed by a neuroradiologist and, independently, by an endocrinologist expert in neuroendocrine imaging: both were blind to the attributed diagnosis. Both investigators were asked to confirm stalk thickening, annotate the dimensions and length of the stalk, evaluate the homogeneity of the thickening, the presence or absence of the posterior hypophyseal bright spot (PHBS), and the MRI characteristics and enhancement pattern (homogeneous/ non-homogeneous) of the adenohypophysis and neurohypophysis. Disagreement was discussed and consensus agreed.

Although the patients were submitted to different protocols, all patients were included in the study on the condition that sagittal and coronal non-enhanced and/ or enhanced T1-weighted images of the hypothalamohypophyseal tract were present; this ultimately led to a collection of 33 T1-weighted pre-contrast and 32 T1-weighted post-contrast images.

Analysis of hypothalamo-hypophyseal region was reviewed at subsequent MRI scans to assess changes occurring throughout the follow-up period. The shape, length and diameter of the pituitary stalk (PS) were measured proximally and distally from sagittal and coronal T1-weighted sequences (14). The stalk was considered enlarged when the diameter was greater than $3.25 \mathrm{~mm}$ in its proximal portion and/or $1.9 \mathrm{~mm}$ in its distal portion, as reported previously (9). The height and depth of the anterior pituitary on the sagittal T1-weighted image, and width on the coronal T1-weighted image, were also recorded for each patient.

Texture analysis (TA) of pituitary and brain MRI was analysed using Image $1.49 \mathrm{v}(\mathrm{NIH})$. The distribution of grey levels between pixels in a given region of interest (ROI) was described by means of the standard deviation (s.D.) of the ROI. Curves were obtained by manually placing the ROI to entirely cover the pituitary stalk (examples are shown in Figs 1 and 2). White (the corpus callosum) and grey brain matter (frontal 


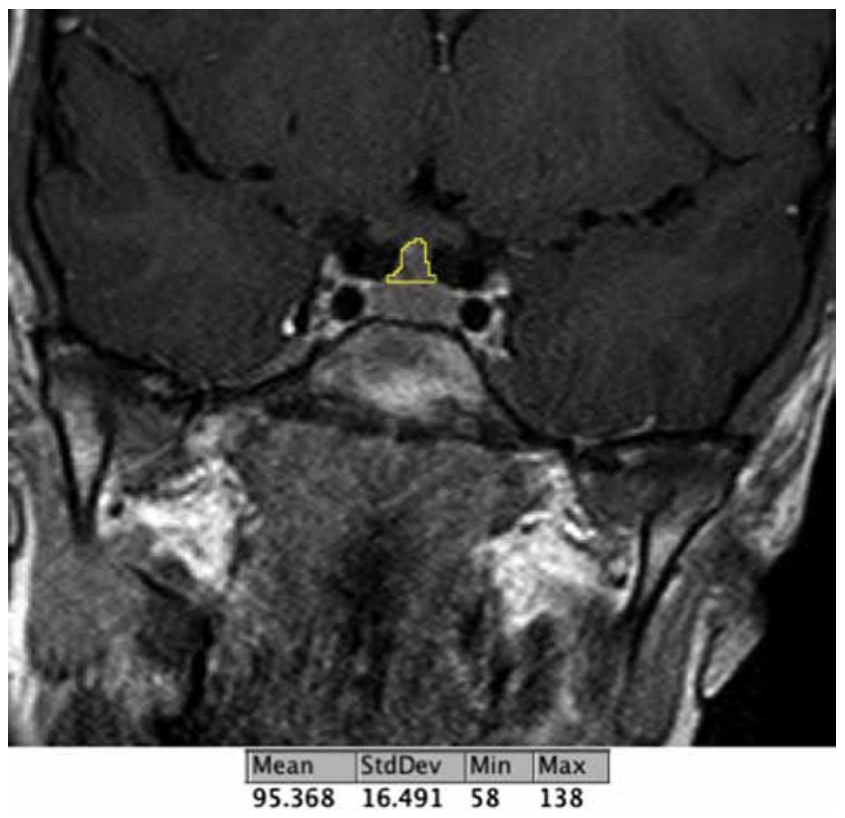

\section{Figure 1}

ImageJ analysis of a pituitary stalk germinoma in a coronal post-gadolinium T1 image with mean grey value, standard deviation (S.D.), minimum and maximum grey value of the circumscribed region of interest (ROI). A full colour version of this figure is available at http://dx.doi.org/10.1530/EJE-16-0455.

cortex circonvolution) intensity and S.D. were used to normalise the data measured at PS levels. Additionally, we measured the texture in the pituitary gland in both sagittal and coronal slices. All parameters were measured in T1-weighted images pre- and post-gadolinium. Two physicians (A M I and E S) independently placed the ROIs and recorded textural parameters. Inter-observer agreement was calculated and, given the substantial reliability for the entire set, data obtained by one operator (E S) were used (Supplementary Tables 1 and 2 , see section on supplementary data given at the end of this article).

\section{Follow-up}

Each patient received a personalised follow-up protocol according to clinical history, symptoms, biochemical and imaging results, but usually consisted of clinical evaluation, endocrine tests, serum tumour markers and pituitary MRI 3 months after diagnosis/surgery and subsequently every 6 months for the first year, and then yearly if the lesion was morphologically and biochemically stable.

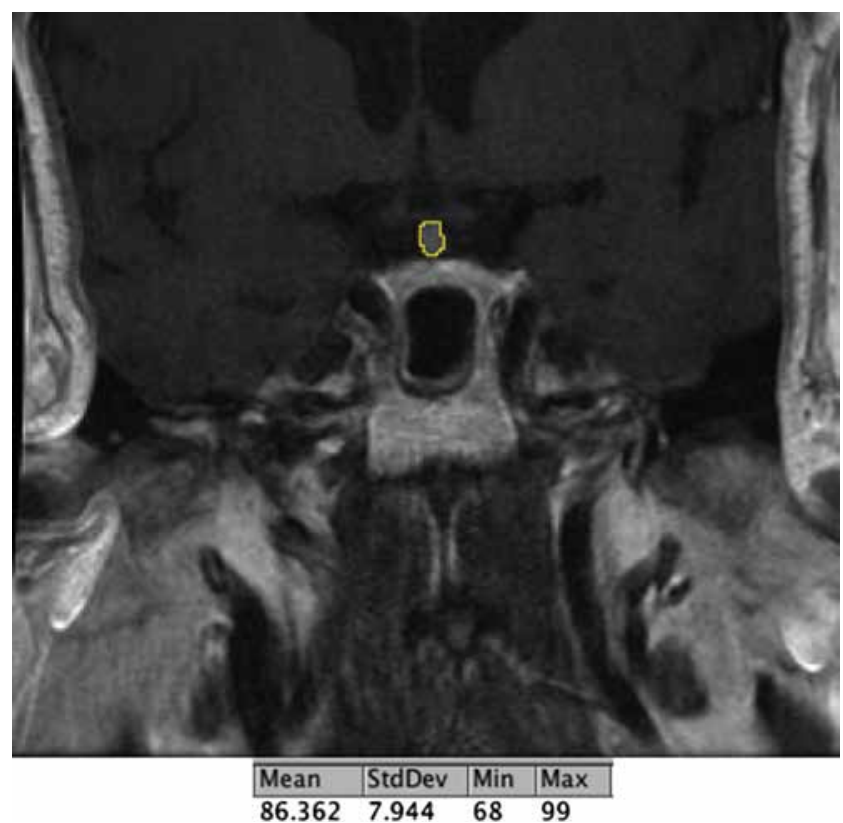

\section{Figure 2}

ImageJ analysis of a non-neoplastic lesion (stable at 36 months follow-up) in a coronal post-gadolinium T1 image with mean grey value, standard deviation (s.D.), minimum and maximum grey value of the circumscribed region of interest (ROI). A full colour version of this figure is available at http://dx.doi. org/10.1530/EJE-16-0455.

\section{Reference diagnosis}

The reference diagnosis was defined on the basis of pathology findings, when available, or on the basis of clinical follow-up. Specifically, non-neoplastic lesions were either histologically proven or responded to initiation/suspension of drugs known to affect pituitary/ pituitary stalk changes, or remained stable or reduced in size on follow-up ( $>12$ months).

We performed a separate texture MRI image analysis comparing neoplastic lesions and just the subgroup of patients with histologically confirmed nonneoplastic lesions (reported in Supplementary Data and Supplementary Table 5).

\section{Data analysis}

We used SPSS (version 17) for statistical analysis of the data. The data presented are mean \pm s.D. As there were two raters for the textural heterogeneity analysis data, the Cohen-k coefficient was used for inter-observer agreement: reliability analysis for quantitative textural parameters was performed with analysis of the inter-class 
correlation coefficients. We performed receiver-operating characteristic (ROC) curves to evaluate the combination of sensitivity and specificity for each possible test positivity cutoff, and we used the area under the ROC curve to express the overall diagnostic accuracy of the index test. $P<0.05$ was considered indicative of a statistically significant difference. STARD guidelines were followed for reporting diagnostic accuracy of this study (15).

This project was registered as an audit (Datix ID number 4062), and registered as such with Oxford University Hospitals Trust.

\section{Results}

\section{Characteristics of the study population}

A total of 42 cases fulfilled the search criteria; of these, 6 were excluded based on the absence of a pre-defined mandatory image slice. Thus, 36 patients (61\% males) with pituitary stalk thickening were included, with a mean age at diagnosis of 37 years (range 4-83, s.D. 22.9).

The nature of pituitary stalk lesion was histologically proven in 18 patients (50\%) and attributed with a high grade of certainty in 4 more patients for a total of 22 patients. Overall, we identified neoplastic lesions in 10 patients (46\%), inflammatory lesions in 7 (32\%) and congenital anomalies in 5 patients (22\%). Of the 10 neoplastic lesions, 2 were considered to be relatively benign: a craniopharyngioma and a pituicytoma. Malignant tumours included six primary germinomas, one Langerhans cell histiocytosis and one secondary lesion from a breast carcinoma. Of inflammatory lesions, three patients had a lymphocytic hypophysitis, one a secondary hypophysitis associated with a ruptured Rathke cleft cyst, and three ipilimumab-induced hypophysitis. With regard to the three patients with ipilimumab-induced hypophysitis, biopsy was not performed, but the diagnosis were considered certain because symptoms, clinical findings and images all improved significantly after drug withdrawal.

Of the congenital lesions, four were Rathke cleft cysts and one PST was considered to be due to ectopic posterior pituitary tissue, as judged by the results of multiple subsequent MRI although, biopsy in this case was not performed.

\section{Diagnosis according to age sub-groups}

In the young patients (<18years), germinoma was diagnosed in 5/10 patients (50\%): one had a craniopharyngioma, one a congenital ectopic posterior pituitary tissue, while three had stable imaging and no new endocrine defect at 2-5 years follow-up.

In the sub-group of middle-age patients (19-59 years), $1 / 20$ patients had a germinoma, one a pituicytoma, one a Langerhans cell histiocytosis and 3/20 (15\%) a Rathke cleft cyst. Interestingly, all seven inflammatory lesions fell in this age category. The remaining $7 / 20$ patients (35\%) did not undergo biopsy and showed stable lesions at follow-up.

In the older age group ( $>60$ years), one had a metastatic adenocarcinoma of breast origin as the first evidence of metastasis after 8 years from the primary diagnosis, while one was diagnosed with a Rathke cleft cyst; four of the six did not undergo surgery and presented stable lesions at 3-5 years of follow-up.

Overall, germinomas were diagnosed at an average age of $12.5 \pm 4.9$ years (range 9-21); 4/6 patients with germinomas had blood tumour markers performed ( $\alpha \mathrm{FP}, \mathrm{hCG}, \mathrm{CEA}$ and ACE) and all were negative, as were the CSF tumour markers in the only patient in whom these were assessed. In 5/6 patients, CSF analysis for cells alone was performed, but in only one case was this diagnostic of germinoma.

With regard to the three patients with ipilimumabinduced hypophysitis, biopsy was not performed, but the diagnosis was considered certain because symptoms, clinical findings and images all improved significantly after drug withdrawal.

\section{Clinical findings and endocrine evaluation}

At the time of diagnosis, DI was present in 17 patients (47\%), headache in 15 patients (42\%), visual problems in 9 (25\%), lethargy in $5(14 \%)$, weight loss in $5(14 \%)$, dizziness in 4 (11\%), poor appetite in $3(8 \%)$ and diminished libido in 3 males (8\%). In three cases (8\%), PST was an incidental finding (Table 1).

Of the 28 patients who had hormonal tests performed at the time of diagnosis, 16/28 (57\%) had at least one anterior pituitary hormonal deficit, while 5/28 (18\%) patients had more than one anterior pituitary axis affected (3 had panhypopituitarism). Secondary hypogonadism was the most common endocrine deficiency (36\%), followed by GH deficiency (21\%), adrenal insufficiency (21\%) and thyroid hormone deficiency (18\%) (Table 2). Two additional patients could not be fully evaluated, presenting to our centre already receiving hydrocortisone replacement therapy. Considering inflammatory lesions alone (7 patients): three showed ACTH deficiency, one did not have ACTH deficiency, while in the remaining three patients, the pituitary-adrenal axis was not assessed. 
Table 1 Description of symptoms on presentation in all patients, patients with neoplastic lesions and patients with non-neoplastic lesions.

\begin{tabular}{|c|c|c|c|}
\hline $\begin{array}{l}\text { Presenting } \\
\text { symptoms }\end{array}$ & $\begin{array}{c}\text { All } \\
(n=36)\end{array}$ & $\begin{array}{c}\text { Neoplastic } \\
\text { lesions } \\
(n=10) \\
\end{array}$ & $\begin{array}{c}\text { Non-neoplastic } \\
\text { lesions } \\
(n=26)\end{array}$ \\
\hline Headache & $15 / 36(41.7 \%)$ & $4 / 10(40 \%)$ & $11 / 26(42.3 \%)$ \\
\hline Visual problems & $9 / 36(25 \%)$ & $3 / 10(30 \%)$ & $6 / 26(23.1 \%)$ \\
\hline Weight loss & $5 / 36(13.9 \%)$ & 2/10 (20\%) & $3 / 26(11.5 \%)$ \\
\hline Lethargy & $5 / 36(13.9 \%)$ & $4 / 10(40 \%)$ & $1 / 26(3.8 \%)^{*}$ \\
\hline Dizziness & $4 / 36(11.1 \%)$ & $1 / 10(10 \%)$ & $3 / 26(11.5 \%)$ \\
\hline Poor appetite & $3 / 36(8.3 \%)$ & $1 / 10(10 \%)$ & $2 / 26(7.7 \%)$ \\
\hline Diminished libido & $3 / 36(8.3 \%)$ & $1 / 10(10 \%)$ & $2 / 26(7.7 \%)$ \\
\hline
\end{tabular}

*Significant difference between neoplastic and non-neoplastic lesions.

Thus, 3/4 (75\%) of inflammatory pituitary stalk lesions patients had ACTH deficiency. Of the 17 patients with DI, 11 also had anterior pituitary hormonal deficiencies.

Comparing the group of patients with neoplasms to the non-neoplastic patients, in the former group, there was a greater prevalence of DI ( $80 \%$ vs $35 \% ; P=0.014)$, secondary hypogonadism $(57 \%$ vs $29 \% ; P=0.03)$, the presence of at least one anterior pituitary deficit (100\% vs $43 \% ; P=0.007)$ and complaint of severe lethargy (40\% vs $4 \% ; P=0.004)$. In particular, the combination of DI plus secondary hypogonadism was a distintintive feature of neoplastic PST (75\% vs 20\%; $P=0.003$, Tables 1 and 2 ).

As a result of surgery, new onset of the following disorders was found: 2 permanent DI, 3 secondary hypogonadism, 10 ACTH deficiency, 3 hypothyroidism, 2 GH deficiency.

During follow-up of non-operated patients, four developed a pituitary deficit that was absent at presentation and the new pituitary deficit occurred after the onset of DI. Specifically, in one patient, hypogonadotrophic hypogonadism developed 1 year after diagnosis as the result of an enlarging lesion, subsequently confirmed as a germinoma. Two patients developed hypogonadotrophic hypogonadism at 1 and 3 years respectively after diagnosis, while one developed GH deficiency 2 years after the onset of DI; however, of the last three patients described, MRI imaging was stable and no biopsy was performed.

Concerning the dimensions of lesions during follow-up, four patients required a pituitary stalk biopsy during follow-up, between 3 and 12 months since first diagnosis, because of an enlargement of the lesions at MRI. All turned out to be neoplastic: two germinomas, one craniopharingioma and one pituicytoma.

\section{MRI characteristics}

Measurements of the stalk on MRI were expressed in terms of length in sagittal and coronal T1-weighted sections, and diameters at the proximal and distal portion of the PS in sagittal and coronal T1-weighted slices (Table 3); height, depth and the width of the anterior pituitary gland are shown in Supplementary Table 3. In MRI imaging, a statistically significant difference in the diameter of neoplastic and nonneoplastic PST was measured at the coronal pre-contrast upper part $(6.3 \pm 3.2$ vs $4.3 \pm 1.6 ; P=0.02)$, sagittal postcontrast upper region $(6.6 \pm 2.5$ vs $4.9 \pm 2.2 ; P=0.04)$ and coronal post-contrast imaging for both upper $(6.4 \pm 3.2$ vs $4.4 \pm 1.7 ; P=0.04)$ and lower regions $(4.6 \pm 2.3$ vs $3.2 \pm 0.9 ; P=0.04)$. Specifically, all lesions above 9.1 $\mathrm{mm}$ in diameter for coronal post-contrast images in the upper part of the stalk and above $5.6 \mathrm{~mm}$ for coronal post-contrast images in the lower part of the stalk were confirmed as neoplastic, achieving 100\% sensitivity. Moreover, all lesions enlarging during follow-up showed neoplasms on histology. Neoplasia was suggested on size criteria or progression with $30 \%$ sensitivity.

Regarding size, inflammatory lesions were also significantly greater than non-inflammatory lesions in terms of height $(8.3 \pm 2.4$ vs $6 \pm 2.2 ; P=0.03)$, depth $(11.3 \pm 0.8$ vs $9.7 \pm 2.3 ; P=0.008)$ and width $(16 \pm 2.8$ vs $11.6 \pm 2.7 ; P=0.002)$ of the pituitary gland (Supplementary Table 3).

Table 2 Distribution of hormonal deficits in all patients, patients with neoplastic lesions and patients with non-neoplastic lesions.

\begin{tabular}{l}
\hline Hormonal deficit \\
\hline Diabetes insipidus (DI) \\
At least 1 anterior pituitary hormonal deficit \\
More than 1 anterior pituitary hormonal deficits \\
Panhypopituitarism \\
Secondary hypogonadism \\
GH deficit \\
ACTH deficit \\
TSH deficit
\end{tabular}

$\begin{array}{r}\hline \text { All }(n=36) \\ \hline 17 / 36(47.2 \%) \\ 16 / 28(57.1 \%) \\ 5 / 28(17.8 \%) \\ 3 / 28(10.7 \%) \\ 10 / 28(35.7 \%) \\ 6 / 28(21.4 \%) \\ 6 / 28(21.4 \%) \\ 5 / 28(17.9 \%) \\ \hline\end{array}$

\begin{tabular}{c}
\hline Neoplastic lesions $(n=10)$ \\
\hline $8 / 10(80 \%)$ \\
$7 / 7(100 \%)$ \\
$2 / 7(28.6 \%)$ \\
$1 / 7(14.3 \%)$ \\
$4 / 7(57.1 \%)$ \\
$2 / 7(28.6 \%)$ \\
$3 / 7(42.9 \%)$ \\
$2 / 7(28.6 \%)$ \\
\hline
\end{tabular}

Non-neoplastic lesions $(n=26)$ $9 / 26(34.6 \%) *$ $9 / 21(42.8 \%) *$ $3 / 21(14.3 \%)$

$2 / 21(9.5 \%)$

$6 / 21(28.6 \%) *$ $4 / 21(19 \%)$ $3 / 21(14.3 \%)$ $3 / 21(14.3 \%)$

*Significant difference between neoplastic and non-neoplastic lesions. 
Table 3 Pituitary stalk diameter characteristics on T1-weighted MRI images comparing all patients, patients with neoplastic lesions and patients with non-neoplastic lesions. Data are presented as mean \pm s.D. (min-max)

\begin{tabular}{l} 
Pituitary stalk diameter characteristics, mm \\
\hline Length sagittal \\
Length coronal \\
Stalk diameter sagittal upper part pre gadolinium \\
Stalk diameter sagittal lower part pre gadolinium \\
Stalk diameter coronal upper part pre gadolinium \\
Stalk diameter coronal lower part pre gadolinium \\
Stalk diameter sagittal upper part post gadolinium \\
Stalk diameter sagittal lower part post gadolinium \\
Stalk diameter coronal upper part post gadolinium \\
Stalk diameter coronal lower part post gadolinium \\
Absence of posterior pituitary bright spot (PPBS)
\end{tabular}

\begin{tabular}{l}
\multicolumn{1}{c}{$\begin{array}{c}\text { All } \\
(n=36)\end{array}$} \\
\hline $7.7 \pm 2.7(3-16)$ \\
$7.3 \pm 2.7(3-14)$ \\
$5.3 \pm 2.4(2.3-1.7)$ \\
$4.6 \pm 2.4(2.3-5.4)$ \\
$4.9 \pm 2.1(2-2)$ \\
$3.6 \pm 1.8(1.5-0.6)$ \\
$5.3 \pm 2.4(1.6-2.1)$ \\
$3.9 \pm 1.3(2-0.6)$ \\
$4.9 \pm 2.3(1.6-2.7)$ \\
$3.6 \pm 1.6(2.2-0.2)$ \\
$24 / 33(72.8 \%)$ \\
\hline
\end{tabular}

Neoplastic lesions $(n=10)$ $8.3 \pm 2.6(5-4)$ $7.9 \pm 2.8(4-3)$ $6.4 \pm 2.7(4-0.6)$ $5.3 \pm 4.5(2.9-5.4)$ $6.3 \pm 3.2(3.6-2)$

$4.6 \pm 2.3(2.2-0.6)$

$6.6 \pm 2.5(4-1.8)$ $3.9 \pm 1.3(2-0.7)$

$6.4 \pm 3.2(3.5-2.7)$

$4.6 \pm 2.3(2.2-0.2)$ $8 / 8(100 \%)$

\begin{tabular}{c}
$\begin{array}{c}\text { Non-neoplastic lesions } \\
(n=26)\end{array}$ \\
\hline $7.4 \pm 2.8(3-16)$ \\
$7.0 \pm 2.7(3-4)$ \\
$5 \pm 2.2(2.3-1.7)$ \\
$4.4 \pm 1.6(2.3-0.9)$ \\
$4.3 \pm 1.6 *(2-0.6)$ \\
$3.4 \pm 1.4(1.5-0.9)$ \\
$4.9 \pm 2.2 *(1.6-2.1)$ \\
$3.8 \pm 1.3(2.1-0.6)$ \\
$4.4 \pm 1.7 *(2.5-0.1)$ \\
$3.2 \pm 0.9 *(2.2-0.6)$ \\
$16 / 25(64 \%)$ \\
\end{tabular}

*Significant difference between neoplastic and non-neoplastic lesions.

There was no direct relationship between the pattern of enhancement and the extent of the lesions. In addition, we were not able to identify any correlation between hypopituitarism and the pattern of enhancement or size of the lesion.

None of patients with neoplastic PST showed the normal PHBS (sensitivity of 100\%), as compared with 64\% of patients with non-neoplastic lesions $(P=0.02)$ (specificity of 36\%). Furthermore, in our population, the simultaneous absence of the PHBS and presence of DI was significantly higher in patients with neoplastic lesions compared with the non-neoplastic group (7/8 (88\%) vs 5/25 (20\%); $P=0.001$ ) with $88 \%$ sensitivity and $80 \%$ specificity.

The MRI patterns of thickening of the stalk in the upper, median and lower part are shown in Supplementary Table 4 . The strongest association with a specific pattern of thickening on cranial MRI was that for congenital lesions; with one exception, 4/5 such lesions showed a thickening of the median part of the stalk. The most frequent diagnosis in this category was a Rathke cleft cyst. Of the inflammatory lesions, 5/7 showed a thickening of the upper part of the stalk, while for neoplastic lesions, 5/10 showed a thickening of the upper part and 5/10 a thickening of the median part of the stalk. In 25 patients (69\%), the abnormal imaging was limited to the pituitary stalk and showed a suprasellar extension in 6 patients (17\%).

\section{Pituitary stalk texture analysis}

Texture analysis (TA) is a software-based image processing that allows to evaluate the distribution of grey levels in a given region of interest (ROI). It provides quantitative data, has been employed in several neurodegenerative disorders and is highly reproducible $(16,17)$. One way to evaluate texture is to measure the standard deviation (s.D.) of the mean grey value of a given region of interest (ROI).

Comparing neoplastic and non-neoplastic PST, texture analysis revealed a significantly higher degree of heterogeneity expressed as standard deviation of the PST in patients with neoplastic lesions compared with non-neoplastic lesions (sagittal pre-gadolinium, $P=0.02$; coronal pre-gadolinium, $P=0.03$; sagittal postgadolinium, $P<0.001$; coronal post-gadolinium, $P<0.001$, Table 4). These findings were confirmed when corrected for the S.D. of white and grey neural tissues.

We performed ROC curve analysis to identify a possible criterion of textural heterogeneity to differentiate neoplastic from non-neoplastic stalk lesions. We found criteria for differentiating these two lesion categories for sagittal pre-gadolinium images of 7.55 s.D. with a sensitivity of $71.4 \%$ and a specificity of $84.6 \%$ (AUC: 0.786, CI: 95\% 0.499-1), coronal pre-gadolinium images of 8.2 s.D. with a sensitivity of $71.4 \%$ and a specificity of $87.5 \%$ (AUC: 0.774 , CI: 95\% 0.499-1), sagittal postgadolinium images of 11.84 s.D. with a sensitivity of $87.5 \%$ and a specificity of $95.8 \%$ (AUC: 0.943 , CI: $95 \%$ 0.832-1; Fig. 1) and coronal post-gadolinium images of 11.61 s.D. with a sensitivity of $88.9 \%$ and a specificity of 91.7\% (AUC: 0.968, CI: 95\% 0.915-1).

\section{Discussion}

In this retrospective analysis, we describe the presenting features, aetiological spectrum, associations between clinical and biochemical data with imaging characteristics, 
Table 4 Pituitary stalk textural heterogeneity characteristics on T1-weighted MRI images comparing all patients, patients with neoplastic lesions and patients with non-neoplastic lesions. Data are presented in millimetres as mean \pm s.D. (min-max)

\begin{tabular}{llc}
\hline Textural heterogeneity & & All $(n=36)$ \\
\cline { 1 - 1 } $\begin{array}{c}\text { Pituitary stalk (sagittal T1 } \\
\text { pre-gadolinium) }\end{array}$ & & $6.572 \pm 2.484(2.877-13.478)$ \\
$\begin{array}{c}\text { Pituitary stalk (coronal T1 } \\
\text { pre-gadolinium) }\end{array}$ & $6.876 \pm 3.923(3.844-3.624)$ \\
$\begin{array}{c}\text { Pituitary stalk (sagittal T1 } \\
\text { post-gadolinium) }\end{array}$ & & $10.909 \pm 5.870(3.305-7.496)$ \\
$\begin{array}{l}\text { Pituitary stalk (coronal T1 } \\
\text { post-gadolinium) }\end{array}$ & & $10.465 \pm 4.233(4.568-4.206)$ \\
\hline
\end{tabular}

histopathology and clinical outcomes of pituitary stalk lesions seen in our university hospital over the last decade. We have also focused on reviewing MRI imaging studies for morphology, signal intensity, enhancement and texture characteristics of the pituitary gland and stalk.

DI was the initial presentation in nearly half of the cases followed by headache, while anterior pituitary dysfunction was frequently present, as reported previously $(6,18)$. However, it is likely that pituitary dysfunction was underestimated, as some of our patients did not have full endocrine evaluation. Secondary hypogonadism was the most common anterior pituitary deficiency, as reported previously (6), followed by deficiency of GH, ACTH/ cortisol and thyroid dysfunction, and in particular 57\% had at least one anterior pituitary hormonal deficit, $18 \%$ patients had more than one anterior pituitary axes affected and $11 \%$ had panhypopituitarism. We found a significantly increased prevalence of DI, secondary hypogonadism, severe lethargy and the presence of at least one anterior pituitary deficit in patients with neoplastic disease compared with the non-neoplastic group, with neoplastic stalk lesions increasing in dimension during the follow-up period. In particular, we recommend more careful evaluation of patients presenting with DI plus secondary hypogonadism because these two conditions were significantly more present in patients with neoplastic lesions.

The absence of the normal PHBS showed 100\% sensitivity but only $36 \%$ specificity in distinguishing between neoplastic and non-neoplastic lesions, while the simultaneous absence of the PHBS and presence of DI showed $88 \%$ sensitivity and $80 \%$ specificity. These results confirmed previous finding reporting that the PHBS is present in most healthy individuals and is absent in many patients with large intrasellar masses and empty sellae, probably due to compression of the posterior lobe (19). However, neither the pattern of MRI enhancement nor

\begin{tabular}{ccc}
\hline Neoplastic lesions $(n=10)$ & & Non-neoplastic lesions $(n=26)$ \\
\hline $8.948 \pm 3.315(4.482-3.478)$ & & $5.932 \pm 1.804 *(2.877-0.178)$ \\
$10.871 \pm 6.462(3.844-3.642)$ & $5.711 \pm 1.704 *(3.889-0.889)$ \\
$18.449 \pm 6.683(9.072-7.496)$ & $8.380 \pm 2.478 *(3.305-2.332)$ \\
$15.660 \pm 4.015(10.046-4.206)$ & $8.517 \pm 2.193 *(4.568-3.440)$ \\
\hline
\end{tabular}

the size of the lesion predicted hypophyseal dysfunction, in keeping with previous data (20).

There are a few published case series on patients with pituitary stalk lesions. Hamilton et al. (21) published the first large case series on pituitary/infundibular lesions including 65 subjects, 44 adults and 21 paediatric patients. Only a small subset of patients (40\%) had their diagnoses proven by histopathology, showing a predominance of neoplastic lesions (37\%), especially in the adult group (21). More recently, Turcu et al., in one of the largest studies on pituitary stalk lesions to date, showed a similar high prevalence of tumour lesions (32\%) with a lower proportion undergoing histopathological confirmation (37/152: 24\%) (6). In our series, neoplastic lesions were the most highly represented category, accounting for $46 \%$ of all patients with certain pathology, possibly related to the higher percentage, and $50 \%$ of patients undergoing a pituitary stalk biopsy. In previous studies, inflammatory lesions comprised 30\% (21) and 20\% of patients (6), not too dissimilar to the $32 \%$ we found. This may be slightly inflated by the inclusion of increasing numbers of patients with hypophysitis secondary to immune checkpoint inhibitors $(22,23,24,25)$. With the recent introduction of these drugs for the treatment of metastatic cancers such as melanoma and their increasing use, it is likely that such patients will be seen more often, although they should not provide difficulty in diagnosis. With regard to congenital lesions, Turcu et al. reported a $9 \%$ prevalence (6), lower compared with that reported in our population of $22 \%$ where there was a predominance of Rathke cleft cysts. However, we confirm the findings from the Mayo Clinic that most congenital pituitary stalk lesions appeared round on MRI.

This study has shown a correlation between stalk diameter dimensions and the presence or absence of neoplastic change, demonstrating that neoplastic stalks are larger compared with non-neoplastic lesions. All 
lesions above $9.1 \mathrm{~mm}$ in diameter for coronal postcontrast images in the upper part of the stalk and above $5.6 \mathrm{~mm}$ for coronal post-contrast images in the lower part of the stalk were neoplastic, and all lesions enlarging during follow-up showed neoplasms on histology.

While previous studies were unable to establish any imaging parameter which would help identify the nature of PST (6), we have evaluated for the first time the textural heterogeneity in terms of intensity of the pituitary stalk with the programme ImageJ. There was a significant correlation between the presence of neoplasia in the pituitary stalk and the standard deviation (s.D.) of the texture of the stalk in sagittal pre- and postgadolinium and in coronal pre- and post-gadolinium T1-weighted images. We have also proposed possible textural heterogeneity criteria for the pituitary stalk for sagittal pre- and post-gadolinium and coronal pre- and post-gadolinium T1 images. These textural heterogeneity criteria were more accurate for post-gadolinium images. ImageJ is a free open-source application to process images, simple to use, and we suggest that this analysis might offer a powerful, cheap and available tool that every experienced neuroendocrinologist could use in clinical practice.

\section{Limitations}

Our study has several limitations: first of all, the small sample, even though collected from a major centre over a prolonged period, with the lack of tissue-confirmed diagnoses in half the cases. Indeed, while in our population the rate of histological confirmation is higher compared with previous studies $(6,21)$, it is probably still insufficient for definitive conclusions. However, one of the main limitations is the retrospective nature of data collection and our own institutional referral bias that may overestimate severe conditions and may underestimate many asymptomatic lesions or lesions with lower clinical impact. Nevertheless, given that performing biopsy of pituitary stalk is a technically demanding technique that has significant risk of morbidity, the diagnosis was frequently based on clinical findings, biochemical evaluation and serial MRI.

In conclusion, the spectrum of pathology involving the pituitary stalk is broad, ranging from indolent to more malignant lesions that can have a dramatically altered prognosis. We suggest that stalk lesions above a certain size, or which enlarge on follow-up, or which show defined textural characteristic, are more likely to require urgent intervention as compared with more indolent lesions best served by a conservative observational approach. It is possible that more sophisticated image analysis as suggested here may expedite attainment of a definitive diagnosis, leading to more rapid and effective therapy.

\section{Supplementary data}

This is linked to the online version of the paper at http://dx.doi.org/10.1530/ EJE-16-0455.

\section{Declaration of interest}

The authors declare that there is no conflict of interest that could be perceived as prejudicing the impartiality of the research reported.

\section{Funding}

This research did not receive any specific grant from any funding agency in the public, commercial or not-for-profit sector.

\section{Acknowledgements}

We thank Dr Giulia Puliani for her advice on ImageJ programme.

\section{References}

1 Maghnie M, Cosi G, Genovese E, Manca-Bitti ML, Cohen A, Zecca S, Tinelli C, Gallucci M, Bernasconi S, Boscherini B et al. Central diabetes insipidus in children and young adults. New England Journal of Medicine 2000343 998-1007. (doi:10.1056/ NEJM200010053431403)

2 Aizer AA, Sethi RV, Hedley-Whyte ET, Ebb D, Tarbell NJ, Yock TI \& Macdonald SM. Bifocal intracranial tumors of nongerminomatous germ cell etiology: diagnostic and therapeutic implications. Neuro-oncology 201315 955-960. (doi:10.1093/neuonc/not050)

3 Ricciuti A, De Remigis A, Landek-Salgado MA, De Vincentiis L, Guaraldi F, Lupi I, Iwama S, Wand GS, Salvatori R \& Caturegli P. Detection of pituitary antibodies by immunofluorescence: approach and results in patients with pituitary diseases. Journal of Clinical Endocrinology and Metabolism 201499 1758-1766. (doi:10.1210/ jc.2014-1049)

4 Di Iorgi N, Allegri AE, Napoli F, Calcagno A, Calandra E, Fratangeli N, Vannati M, Rossi A, Bagnasco F, Haupt R et al. Central diabetes insipidus in children and young adults: etiological diagnosis and long-term outcome of idiopathic cases. Journal of Clinical Endocrinology and Metabolism 201499 1264-1272. (doi:10.1210/jc.2013-3724)

5 Di Iorgi N, Morana G \& Maghnie M. Pituitary stalk thickening on MRI: when is the best time to re-scan and how long should we continue re-scanning for? Clinical Endocrinology $201583449-455$. (doi:10.1111/cen.2015.83.issue-4)

6 Turcu AF, Erickson BJ, Lin E, Guadalix S, Schwartz K, Scheithauer BW, Atkinson JL \& Young WF Jr. Pituitary stalk lesions: the Mayo Clinic experience. Journal of Clinical Endocrinology and Metabolism 201398 1812-1818. (doi:10.1210/jc.2012-4171)

7 Guzzo MF, Bueno CB, Amancio TT, Rosemberg S, Bueno C, Arioli EL, Glezer A \& Bronstein MD. An intrasellar germinoma with normal tumor marker concentrations mimicking primary lymphocytic hypophysitis. Arquivos Brasileiros de Endocrinologia e Metabologia 2016 $\mathbf{5 7} 566-570$. 
8 Khare S, Jagtap VS, Budyal SR, Kasaliwal R, Kakade HR, Bukan A, Sankhe S, Lila AR, Bandgar T, Menon PS et al. Primary (autoimmune) hypophysitis: a single centre experience. Pituitary 201518 16-22. (doi:10.1007/s11102-013-0550-9)

9 Simmons GE, Suchnicki JE, Rak KM \& Damiano TR. MR imaging of the pituitary stalk: size, shape, and enhancement pattern. American Journal of Roentgenology 1992159 375-377. (doi:10.2214/ ajr.159.2.1632360)

10 Caturegli P, Newschaffer C, Olivi A, Pomper MG, Burger PC \& Rose NR. Autoimmune hypophysitis. Endocrine Reviews 200526 599-614. (doi:10.1210/er.2004-0011)

11 Kaltsas GA, Powles TB, Evanson J, Plowman PN, Drinkwater JE, Jenkins PJ, Monson JP, Besser GM \& Grossman AB. Hypothalamopituitary abnormalities in adult patients with langerhans cell histiocytosis: clinical, endocrinological, and radiological features and response to treatment. Journal of Clinical Endocrinology and Metabolism 200085 1370-1376. (doi:10.1210/jc.85.4.1370)

12 Janmohamed S, Grossman AB, Metcalfe K, Lowe DG, Wood DF, Chew SL, Monson JP, Besser GM \& Plowman PN. Suprasellar germ cell tumours: specific problems and the evolution of optimal management with a combined chemoradiotherapy regimen. Clinical Endocrinology 200557 487-500.

13 Rupp D \& Molitch M. Pituitary stalk lesions. Current Opinion in Endocrinology, Diabetes, and Obesity 200815 339-345. (doi:10.1097/ MED.0b013e3283050844)

14 Satogami N, Miki Y, Koyama T, Kataoka M \& Togashi K. Normal pituitary stalk: high-resolution MR imaging at 3T. American Journal of Neuroradiology 201031 355-359. (doi:10.3174/ajnr.A1836)

15 Bossuyt PM, Reitsma JB, Bruns DE, Gatsonis CA, Glasziou PP, Irwig L, Lijmer JG, Moher D, Rennie D, de Vet HC et al. STARD 2015: an updated list of essential items for reporting diagnostic accuracy studies. Clinical Chemistry 201561 1446-1452. (doi:10.1373/ clinchem.2015.246280)

16 Doan NT, van den Bogaard SJ, Dumas EM, Webb AG, van Buchem MA, Roos RA, van der Grond J, Reiber JH \& Milles J. Texture analysis of ultrahigh field T2*-weighted MR images of the brain: application to Huntington's disease. Journal of Magnetic Resonance Imaging 201439 633-640. (doi:10.1002/jmri.24199)
17 de Albuquerque M, Anjos LG, Maia Tavares de Andrade H, de Oliveira MS, Castellano G, Junqueira Ribeiro de Rezende T, Nucci A \& Cavalcante Franca Junior M. MRI texture analysis reveals deep gray nuclei damage in amyotrophic lateral sclerosis. Journal of Neuroimaging 201626 201-206. (doi:10.1111/jon.2016.26.issue-2)

18 Werny D, Elfers C, Perez FA, Pihoker C \& Roth CL. Pediatric central diabetes insipidus: brain malformations are common and few patients have idiopathic disease. Journal of Clinical Endocrinology and Metabolism 2015100 3074-3080. (doi:10.1210/jc.2015-1287)

19 Colombo N, Berry I, Kucharczyk J, Kucharczyk W, de Groot J, Larson T, Norman D \& Newton TH. Posterior pituitary gland: appearance on MR images in normal and pathologic states. Radiology 1987165 481-485. (doi:10.1148/radiology.165.2.3659370)

20 Leger J, Velasquez A, Garel C, Hassan M \& Czernichow P. Thickened pituitary stalk on magnetic resonance imaging in children with central diabetes insipidus. Journal of Clinical Endocrinology and Metabolism 199984 1954-1960. (doi:10.1210/jc.84.6.1954)

21 Hamilton BE, Salzman KL \& Osborn AG. Anatomic and pathologic spectrum of pituitary infundibulum lesions. American Journal of Roentgenology 2007188 W223-W232. (doi:10.2214/ajr.05.2027)

22 Spain L, Diem S \& Larkin J. Management of toxicities of immune checkpoint inhibitors. Cancer Treatment Reviews 201644 51-60. (doi:10.1016/j.ctrv.2016.02.001)

23 Dillard T, Yedinak CG, Alumkal J \& Fleseriu M. Anti-CTLA-4 antibody therapy associated autoimmune hypophysitis: serious immune related adverse events across a spectrum of cancer subtypes. Pituitary $2010 \mathbf{1 3}$ 29-38. (doi:10.1007/s11102-009-0193-z)

24 Juszczak A, Gupta A, Karavitaki N, Middleton MR \& Grossman AB. Ipilimumab: a novel immunomodulating therapy causing autoimmune hypophysitis: a case report and review. European Journal of Endocrinology/European Federation of Endocrine Societies 2012 167 1-5.

25 Albarel F, Gaudy C, Castinetti F, Carre T, Morange I, ConteDevolx B, Grob JJ \& Brue T. Long-term follow-up of ipilimumabinduced hypophysitis, a common adverse event of the anti-CTLA-4 antibody in melanoma. European Journal of Endocrinology/European Federation of Endocrine Societies 2015172 195-204. (doi:10.1530/EJE14-0845)

Received 26 May 2016

Revised version received 2 July 2016

Accepted 14 July 2016 